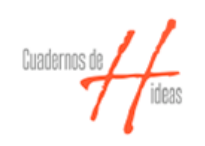

Cuadernos de $\mathrm{H}$ ideas

ISSN: 2313-9048

cuadernosdehideas@perio.unlp.edu.ar

Universidad Nacional de La Plata

Argentina

\title{
Una mirada ampliada sobre la precariedad post-industrial. El caso de los trabajadores informáticos a partir de estudios sociales recientes
}

\author{
Adamini, Marina \\ Una mirada ampliada sobre la precariedad post-industrial. El caso de los trabajadores informáticos a partir de \\ estudios sociales recientes \\ Cuadernos de $\mathrm{H}$ ideas, vol. 13, núm. 13, e021, 2019 \\ Universidad Nacional de La Plata, Argentina \\ DOI: https://doi.org/10.24215/23139048e021
}

Esta obra está bajo una Licencia Creative Commons Atribución-NoComercial-Compartirlgual 4.0 Internacional. 
Artículos

\title{
Una mirada ampliada sobre la precariedad post-industrial. El caso de los trabajadores informáticos a partir de estudios sociales recientes
}

\author{
An extended look at post-industrial precariousness. The case of \\ computer workers from recent social studies. \\ Marina Adamini marinaadamini@gmail.com \\ Comisión Nacional de Investigaciones Científicas y Técnicas - \\ Universidad Nacional de Centro, IGEHCS-IHES, Argentina
}

Recibido: 12/06/19

Aceptado: 24/07/19

Publicado: 18/09/19

DOI: https://doi.org/10.24215/23139048e021
Resumen: Realizamos un análisis ampliado de la precarización laboral en el marco de la sociedad post-industrial, a partir del caso del sector de software y servicios informáticos. Éste resulta uno de los nuevos sectores con mayor crecimiento comercial, pero desregulado y segmentado en sus condiciones laborales. Nuestro propósito es analizar la precarización que atraviesa este sector emblemático de la sociedad postindustrial, tomando en consideración no sólo sus condiciones contractuales sino también las subjetivas y de las relaciones laborales, a partir de estudios recientes y fuentes estadísticas.

Palabras clave: precariedad laboral, sociedad post-industrial, sector de software y servicios informáticos, Argentina .

Abstract: We perform an extended analysis of precarious work in the context of the post-industrial society, from the case of software and IT services industry. This is one of the new sectors with greater commercial growth, but deregulated and segmented in their working conditions. Our purpose is to analyze the precariousness that crosses this emblematic sector of post-industrial society, taking into accounts not only its contractual conditions but also the subjective and the labor relations, based on recent studies and statistical sources.

Keywords: Precarious work, post-industrial society, software and computer services sector, Argentina .

\section{Introducción}

El estudio de la precariedad laboral como problemática emerge a mediados de los años 70 en el marco del avance y consolidación de la ofensiva neoliberal sobre el mundo del trabajo, que propagó nuevas formas laborales inestables y desprotegidas. La génesis de este concepto implicaba un propósito de denuncia sobre el proceso de degradación laboral que atravesaban las condiciones de estabilidad, seguridad y protección laboral vigentes en los treinta años gloriosos del modelo industrial asalariado fordista (1945-1973). Desde una perspectiva dicotómica, el empleo precario era definido como una nueva forma laboral inestable, insegura y desprotegida, cuyo principal indicador resultaba la condición de no registro de la relación contractual. 
La consolidación de la precarización laboral como forma-empleo durante los años 90 y 2000, condujo a las Ciencias Sociales del Trabajo a incorporar nuevas dimensiones de análisis sobre la precarización laboral, en torno a sus implicancias en la subjetividad y en las relaciones laborales de los trabajadores. Sin embargo, en términos generales, la precarización laboral continúa siendo definida (y medida) en las Ciencias Sociales del Trabajo como el empleo en negro (no registrado), principalmente por su factibilidad para la operacionalización estadística, como indicador de otras desprotecciones e inestabilidades estrictamente contractuales.

El objetivo de este artículo consiste en proponer un instrumento de análisis de la precarización laboral en el marco de la sociedad post-industrial desde una mirada ampliada, tomando en consideración no sólo sus formas contractuales sino también proponiendo una operacionalización de las dimensiones subjetivas y las relaciones laborales que conforman al mundo del trabajo. Dicha propuesta de abordaje ampliado se aplicará en un estudio de caso instrumental basado en el sector de software y servicios informáticos (SSI), que resulta un sector emblemático de la sociedad post-industrial caracterizado por el uso intensivo de la tecnología y el conocimiento, la desterritorialización y globalización productiva, y la aplicación de formas laborales basadas en la subcontratación y el empleo free lance.

Nuestro supuesto es que a pesar de que la sociedad postindustrial implica una nueva etapa en la organización productiva, mantiene problemáticas laborales históricas respecto a la desprotección e inestabilidad de los trabajadores. La indagación de dichas problemáticas implica asumir una mirada que exceda la dimensión contractual tradicional de abordaje en los estudios del trabajo. Esto implica observar la degradación laboral también en las insatisfacciones de los trabajadores respecto a su trabajo y en su debilitamiento como colectivo de trabajadores, en el marco de las relaciones de fuerza capital-trabajo. De forma concreta, nuestra propuesta en este artículo consiste en delinear los principios teóricos para un análisis ampliado de la precariedad laboral que contemple no sólo las condiciones contractuales del empleo sino también la subjetividad de los trabajadores y sus relaciones laborales.

Particularmente, pensar la precarización laboral en el sector de SSI implica un proceso de problematización de uno de los nuevos sectores productivos basados en el conocimiento que es considerado de los más pujantes en la economía argentina contemporánea. Sin negar su potencialidad productiva y comercial (que resulta creciente no sólo en nuestro país sino a nivel mundial), buscamos dar luz a las ambivalencias laborales que se dan en la desregulación y segmentación laboral del sector. Dicha situación se encuentra propiciada, principalmente, por la ausencia de un Convenio Colectivo de Trabajo (CCT) que normativice y regule las condiciones laborales de los Trabajadores Informáticos (TI).

El estudio de la precariedad laboral en el sector de SSI resulta un campo de estudios en construcción en las Ciencias Sociales del Trabajo, debido a la novedad del sector y también a su creciente pujanza productiva que muchas veces desdibuja la problematización de sus condiciones laborales, 
que se suponen en sintonía con dicha bonanza económica. A pesar de ello, en los últimos años, se dieron aportes en su estudio a partir de los trabajos de Montes Cató (2010, 2011), Rabosto y Zukerfeld (2017), Míguez (2017), Del Fueyo (2017) y Palermo (2018), entre otros, que advierten acerca de diferentes problemáticas laborales de los TI, ligadas a la deslaboralización e individualización, la tercerización, los bajos salarios, la falta de reconocimiento sindical, entre otras.

En términos metodológicos, vamos a realizar un análisis de contenido (Berelson, 1952) de fuentes bibliográficas sobre estudios recientes de las Ciencias Sociales del Trabajo que aludan a las condiciones de trabajo en el sector de SSI, problematizándolas desde nuestro codificación teórica ampliada de la precarización laboral. En complemento con ello, analizaremos fuentes estadísticas del sector, provenientes de Observatorio de Empleo y Dinámica Empresarial (OEDE) -de la Secretaría de Empleo y Trabajo de la Nación de la Nación- y del Observatorio Permanente de la Industria de Software y Servicios Informáticos (OPSSI) de la Cámara de Empresas de Software y Servicios Informáticos (CESSI)-, para su contextualización sectorial.

El artículo se organiza en tres partes, una primera donde se presenta nuestra propuesta teórica de análisis ampliado de la precariedad laboral, incluyendo allí la caracterización de su dimensión contractual, subjetiva y de relaciones laborales; una segunda parte dedicada a la caracterización del sector de SSI en el marco de la sociedad post-industrial y la Argentina contemporánea, en términos económicos y laborales; y una última parte en donde aplicamos nuestra concepción teórica ampliada de la precariedad laboral en el abordaje de estudios sociales recientes del sector de SSI, codificados analíticamente a partir de las tres dimensiones señaladas.

\section{Precariedades: hacia un abordaje ampliado de la degradación de las condiciones laborales}

El concepto de precariedad laboral surge en la década del 70, en el campo académico y en organismos oficiales, como una forma de denuncia de las nuevas formas laborales flexibles que comenzaban a expandirse en el marco de la ofensiva neoliberal. En su origen, se trataba de una categoría que se definía por oposición al modelo de empleo vigente durante los 30 años gloriosos de la posguerra (1945-1973), en la llamada vigencia de la sociedad salarial caracterizada por la estabilidad, protección y seguridad laboral. Si bien aún hoy resulta objeto de cuestión el nivel de generalidad que tuvo esta forma de empleo, sobre todo en regiones como la latinoamericana (Cavarozzi, 1996; Garretón, 2002), el concepto de precariedad se expandió como forma de describir las nuevas formas de empleo inestables y desprotegidas (muchas de ellas legitimadas legalmente) construidas durante el post-fordismo.

Esta primera definición de la precariedad laboral tenía un fuerte contenido normativista (respecto a lo que el empleo debe ser, considerado por la OIT como empleo decente) y un abordaje de tipo jurídico- 
contractual de la problemática. Es decir, se pensaba como precario al empleo en negro (no registrado ${ }^{1}$ ), que privaba a los trabajadores del acceso a derechos, estabilidades y protecciones propias de una relación asalariada formal. En sintonía con ella comenzaron a hacerse las primeras mediciones, en donde la ausencia de aportes al sistema de seguridad social era considerada su principal indicador. Esta definición, surgida en los países centrales, se expandió en los años 80 a nuestra región latinoamericana, donde diferentes estudios sociales se dedicaron a medir la precariedad del empleo a partir de esta perspectiva contractual y normativista.

En Argentina, se destaca el trabajo de Cynthia Pok (1992) que identifica como trabajadores precarios a aquellos que cuentan con

\begin{abstract}
una inserción endeble en el sistema productivo, resaltando su perspectiva jurídica al señalar que la precariedad se refleja en la existencia de condiciones contractuales que no garantizan la permanencia de la relación de dependencia (contratos de tiempo parcial, eventual y demás modalidades restringidas, no sujeción a la percepción de indemnización por despido, etc.) así como en el desempeño en ocupaciones en vías de desaparición o de carácter redundante en términos de necesidades del aparato productivo (Pok, 1992, p. 10).
\end{abstract}

Durante los años 90, la consolidación del modelo neoliberal y de la flexibilidad laboral propició nuevas reflexiones en torno a la precarización laboral, fundamentalmente en torno a sus implicancias sobre la subjetividad de los trabajadores. Se expandieron así estudios sociales que comenzaban a hablar de los riesgos de la precariedad laboral sobre las identidades, advirtiendo un proceso de extrañamiento del trabajador con el trabajo como espacio de sociabilidad y constitución subjetiva, y la dificultad que esto implicaba en las posibilidades de organización colectiva.

Este proceso se describía a partir de metáforas como desafiliación (Castel, 1997), identidades fluidas (Bauman, 2000), corrosión del carácter (Sennett, 2000), crisis de las identidades (Dubar, 2002), entre otras.

En ese marco, desde la Sociología francesa, autores neodurkhemianos sugirieron incorporar a la medición de las condiciones contractuales de la precariedad, el análisis de su dimensión subjetiva. Se destaca allí el trabajo de Serge Paugam (2000) que sumó como nuevo indicador de la precariedad a la insatisfacción del trabajador ante la ausencia de reconocimiento material y/o simbólico de sus tareas. Para el autor, el trabajo y el empleo constituyen las dos dimensiones de análisis de la integración laboral. Define así la precariedad del empleo a partir de las desprotecciones contractuales y la precariedad del trabajo a partir de la falta de reconocimiento del trabajador, su sensación de falta de valoración por el otro, expresada de forma material y simbólica en diferentes circunstancias: bajos salarios, asignación de tareas sin interés, intensidad del trabajo, tensas relaciones sociales con colegas y superiores, entre otras.

Durante los 2000, se destacan nuevos aportes en el abordaje de la precariedad desde la escuela neormaxista francesa, con los trabajos de Sophie Béroud y Paul Bouffartigue (2009) y Patrick Cingolani (2009), 
quienes analizan la precarización laboral en el marco de las relaciones de fuerza entre capital y trabajo. Así, estos autores incorporan al estudio de la precarización una tercera dimensión ligada a las relaciones laborales, que se expresa en una débil aplicación de sus derechos sindicales, lo cual segmenta a los trabajadores y dificulta el desarrollo de herramientas de lucha conjuntas. Señalan al respecto que la precarización favorece el desarrollo de un asalariado bridé (maniatado) (Moulier-Boutang, 1998), dificultado (aunque no imposibilitado) sus formas de reacción y organización colectiva.

En ese sentido no piensan a la precariedad como un estado (precario/ no precario) que corresponde a un contrato o a la subjetividad de un trabajador determinado, sino que se la concibe de forma relacional, como un proceso que atraviesa al colectivo de trabajadores (Appay, 2005) dificultando su organización y representación colectiva. Aunque destacan también que, en términos dialécticos, ella obtiene como respuesta resistencias de los trabajadores, que deben ser también consideradas en el estudio del proceso de precarización.

A partir de estos aportes, definimos a la precariedad como una ofensiva que degrada las condiciones laborales, a partir de la extensión de la inestabilidad y desprotección contractual; la falta de reconocimiento material y simbólico de las tareas; y la débil aplicación de los derechos sindicales de trabajo. Esto implica pensarla como una degradación de las condiciones laborales que afecta a los trabajadores no sólo en sus condiciones de contratación, sino también en su subjetividad y en sus relaciones laborales. En términos concretos esta definición ampliada de la precariedad laboral incluye tres dimensiones analíticas, que refieren respectivamente a sus distintas unidades de observación:

1. dimensión contractual: referida a la inestabilidad y ausencia de protecciones laborales (reconocimiento jurídico de la relación laboral, contrato por tiempo indeterminado, obra social, aportes jubilatorios, indemnización por despido, asignaciones familiares, licencias, etc.);

2. dimensión subjetiva: referida a la insatisfacción de los trabajadores ante la falta de reconocimiento simbólico (en la asignación de tareas, tipo e intensidad de trabajo) y material de su trabajo (salarios, aumentos, premios);

3. dimensión de relaciones laborales: referida a la débil aplicación de sus derechos sindicales en términos formales (ausencia de derecho a la afiliación sindical, ausencia de sindicato reconocido) y de hecho (falta de representatividad sindical, hostigamiento empresarial a la práctica sindical).

A partir del abordaje ampliado de la precarización laboral es posible encontrar diversas situaciones laborales degradadas que combinan de diferentes maneras las dimensiones señaladas. A modo de ejemplo, podemos encontrar en el sector público a trabajadores que cuentan con contratos estables, seguros y protegidos, con el cumplimiento efectivo de sus derechos sindicales, pero que sin embargo encuentran insatisfacción 
en su tarea por realizar tareas mecánicas y burocráticas que carecen de su interés (Diana Menéndez, 2009). Por otro lado, y de modo contrario, podemos encontrar en el periodismo y la industria cultural a trabajadores que encuentran satisfacción e interés en sus tareas pero que, sin embargo, cuentan con empleos totalmente desprotegidos e inestables, muchas veces sin régimen contractual que lo regule o de modo free lance, ajenos a derechos sindicales de representación (Henry, 2013; Bulloni, 2013).

Esta definición ampliada de la precariedad se encuentra amparada en una perspectiva (también) ampliada del trabajo (De la Garza, 2010), en donde se lo considera, además de un medio de acceso a medios materiales y protecciones sociales, como una actividad de realización humana inserta en un conjunto de relaciones sociales de solidaridad y también de conflicto. Se trata por tanto de una definición que ilumina la degradación de las condiciones laborales más allá del empleo, lo cual habilita a pensar la precarización laboral incluso en aquellas formas laborales asalariadas y registradas, como es el caso del sector de SSI. Para ello se incluyen dimensiones de análisis de la precariedad alternativas a la contractual, como son las subjetiva y de relaciones laborales, abordándolas en tanto elementos constitutivos y articulados de los lugares laborales.

Si bien este abordaje ampliado de la precariedad cuenta como potencialidades la posibilidad de detectar situaciones de precariedad laboral heterogéneas e invisibilizadas en empleos formales, tiene como principal limitación la dificultad de su medición estadística. Esta limitación se ampara en la aplicación de la metodología cualitativa necesaria para abordar en profundidad las subjetividades de los trabajadores y su entramado de relaciones laborales. Lo que conduce a que la mayoría de los trabajos donde se aplican una concepción ampliada de la precariedad sean estudios de caso, enmarcados contextual e históricamente (Longo y Busso, 2017; Longo, 2014; Battistini, 2009; Diana Menéndez, 2007, 2009; Adamini, 2014), que muchas veces dificulta la generalización y comparación de sus resultados.

\section{Sector del SSI, un gigante productivo emblema de la sociedad post-industrial}

El sector de software y servicios informáticos (SSI) resulta un sector productivo novedoso y característico de la sociedad post-industrial, que ha atravesado un pujante crecimiento comercial y laboral en los últimos años. Abarca como actividades al desarrollo de productos de software y la provisión de servicios informáticos, que son aplicados al resto de los sectores productivos y también en la propia cotidianidad del mundo de la vida (en computadoras personales, celulares, artefactos electrónicos, etc.). Si bien no siempre es posible marcar una distinción entre ambas actividades, dentro de los productos de software se incluye la elaboración de programas, aplicaciones, mecanismos de seguridad informática, plataformas educativas, etc., mientras que los servicios informáticos incluyen el mantenimiento, aplicación y soporte de los 
mismos, además de la capacitación, consultoría informática y desarrollo web, entre otros (López y Ramos, 2013).

El proceso de continuo crecimiento del sector en los últimos 15 años en Argentina se vio favorecido por diferentes factores, entre ellos podemos mencionar la expansión del capitalismo informacional a nivel global, las condiciones macroeconómicas nacionales tras la devaluación cambiaria a inicios de los años 2000, y las políticas fiscales de incentivos al desarrollo sectorial por parte del Estado Nacional desde 2003 (que continúan vigentes). En este periodo, se duplicó el número de empresas y de trabajadores (Nahirñak, 2006), no sólo en los grandes centros urbanos como Buenos Aires, Rosario y Córdoba, sino también en clusters regionales como Mendoza, Bahía Blanca, Mar del Plata y Tandil, entre otros.

Juan Montes Cató (2010) señala que el crecimiento de la industria de SSI debe entenderse en el marco de consolidación de una nueva etapa del capitalismo, tras la crisis del paradigma productivo fordista, a mediados de los 70. Ésta crisis implicó cambios no sólo en el patrón de organización productiva sino también en las relaciones laborales, donde la tecnología comenzó a jugar un rol central. En ese sentido, el autor habla de la expansión de una fábrica difusa y de nuevas formas laborales flexibles bajo modalidades contractuales descentralizadas como la tercerización y el empleo freelance. Frente a la hegemonía de la actividad industrial y el empleo asalariado, estable y protegido de la etapa fordista, se habla del pasaje a un capitalismo cognitivo (Boutang, 1999) basado en el trabajo inmaterial, bajo la forma temporal e independiente, donde la informática gana lugar no sólo en una herramienta de comunicación e integración global sino también de producción a distancia.

En sintonía con ello, diversos autores (Míguez, 2010; Novick et al, 2004; Mochi Alemán, 2002) coinciden en señalar a las empresas del sector de SSI como emblemas del modelo productivo post-industrial, por el rol que cumple en ella el conocimiento como elemento de producción y las nuevas formas que asume la organización del trabajo, basados en un esquema más horizontal, con equipos de trabajo y proyectos temporales, que se contrapone al modelo industrial, vertical y de producción en masa del modelo fordista industrial.

En términos de formas laborales, frente al modelo fordista de empleo asalariado y estable, se expanden nuevas formas de contratación basadas en prestación temporal de servicios, el uso intensivo de tecnologías de comunicación y esquemas de tercerización de tareas, que amplían las fronteras de las fábricas, incluyendo la posibilidad de encontrar dentro del sector de SSI a equipos trabajando de forma remota desde sus casas y en diferentes países. La contratara de esta flexibilización del trabajo postindustrial es la desprotección a la que se encuentran expuestos los TI en un marco de deslaboralización e inestabilidad de sus contratos (Montes Cató, 2010), situación que abordaremos en profundidad en el próximo aparatado de análisis de precarización laboral en el sector SSI.

En el caso de Argentina, el desarrollo del sector de SSI tuvo sus raíces entre los años 50 y 70, bajo el modelo de industrialización 
por sustitución de importaciones (Borello et al., 2004; Babini, 2003; Azpiazu, Basualdo y Nochteff, 1990) que promovió el desarrollo de instituciones científicas ligadas a lo que luego se consolidaría como la industria informática. El golpe de militar de 1976 y la instauración del modelo económico neoliberal aperturista desalentaron la continuidad de esta política institucional, dejando el crecimiento del sector a la deriva de la espontaneidad de sus agentes privados. Si bien en los años 90, la política de convertibilidad cambiaría favoreció el ingreso de tecnología importada y el desarrollo de servicios informáticos ligados a su instalación y mantenimiento, no había incentivos estatales para el desarrollo productivo de una industria nacional del conocimiento. Fue recién a partir del 2003, con el desarrollo estatal de políticas específicas y la devaluación monetaria, que el sector de SSI tomó un fuerte impulso de crecimiento continuo en Argentina, con instalación de empresas internacionales, creación de múltiples empresas nacionales y fuerte crecimiento de sus ventas y exportaciones.

Respecto a las políticas estatales de fomento al sector instauradas durante el gobierno de Néstor Kirchner (2003-2007) se destacan la Ley No 25.856, sancionada en 2003, que declaró a la producción de software como actividad industrial y por tanto habilitó su acceso a beneficios impositivos y crediticios destinados a dicha actividad, y la Ley No 25.922 de Promoción de la industria del software ${ }^{2}$, sancionada en 2004, que estableció un régimen fiscal especial para empresas del sector por diez años (incluyendo reducción de aportes patronales y estabilidad fiscal), que se extendió hasta fines de $2030^{3}$. Durante el actual gobierno de Mauricio Macri (2015-continua) se mantuvieron vigentes estas políticas de fomento al sector (al cual se considera estratégico) y se incorporó la creación de la Subsecretaría de Servicios Tecnológicos y Productivos ${ }^{4}$ , en el área del Ministerio de Producción, orientada al desarrollo del sector de servicios tecnológicos, fomento de inversiones, articulación de programas de financiamiento y diseño de políticas educativas orientadas a la formación de recursos humanos para el sector (Ministerio de Hacienda y Finanzas Públicas Presidencia de la Nación, 2016).

Durante estos 16 años de crecimiento productivo del sector de SSI en Argentina hubo un correlato en el aumento de la cantidad de empresas, ventas (sobre todo las exportaciones, que resultaban marginales hasta entonces) y número de trabajadores (López y Ramos, 2008). Según el OEDE del MTEySS, entre 2006 y 2015 el número de empresas creció casi un 50\%, dándose la mayor intensidad en la primera etapa (hasta 2011). Respecto a las ventas, se dio un cambio de orientación hacia la exportación: mientras en el 2000 el sector de SSI sólo exportaba el 13\% de su facturación (Bekerman \& Cataife, 2001), en 2008 la proporción era casi del 60\% (frente el 30\% del promedio de PyMEs industriales) ${ }^{5}$ (OPSSI, 2008).

En términos de empleo, el SSI es la rama que más creció en empleo registrado entre 1997 y 2017, teniendo una variación de 16 mil empleos en 1997 a 97 mil en 2017) (Fuente: OEDE-MTEySS). Esta tendencia de crecimiento en la cantidad de trabajadores se mantiene en ascenso. 
Según el último informe del Observatorio Permanente de la Industria de Software y Servicios Informáticos de la Argentina (OPSSI), el empleo en el sector creció un $42,2 \%$ entre 2008 y 2017 , a una tasa anual acumulativa del $4 \%$, año en el cual estiman que la cantidad de empleados sería de unos 107 mil, considerando también a los trabajadores no registrados ${ }^{6}$ (OPSSI, 2018).

En términos descriptivos, diferentes autores (Montes Cató, 2010; López y Ramos, 2013) caracterizan al sector de SSI en Argentina constituido por tres grandes tipo de empresas: 1) un pequeño número de empresas grandes, mayoritariamente de capital extranjero, dedicadas a la comercialización de productos extranjeros y de servicios informáticos para grandes clientes; 2) un conjunto, también pequeño, de empresas nacionales medianas, que desarrollan software y dan servicios informáticos; y 3) un gran número de pequeñas y heterogéneas empresas nacionales, muchas de ellas nuevas, que se dedican al desarrollo de software local y a proveer servicios informáticos diversos, insertándose en redes de subcontratación de servicios de grandes empresas internacionales.

Pero por fuera de dicha clasificación empresarial se encuentra una importante y desconocida cantidad de informáticos trabajando bajo la modalidad free lance, en forma independiente y remota desde sus casas para distintos clientes (Rabosto y Zukerfeld, 2017). Resulta recurrente en el sector el pasaje de una relación asalariada a otra informal de tipo free lance, propiciada por la facilidad de trabajo remoto que habilita la tecnología, ya que sólo se necesita de una computadora y conexión a internet como capital físico (Novick et al, 2011; López y Ramos, 2013).

En diferentes informes de OPSSI se señala que el principal objeto de disputa en el sector resulta el capital humano, siendo la falta de personal capacitado una demanda constante de los empresarios del sector (López y Ramos, 2009). Como respuesta se impulsaron desde cámaras empresariales y desde el Estado Nacional diferentes políticas de capacitación para la fuerza de trabajo del sector, entre las que se destaca el reciente plan $111 \mathrm{mil}$, aplicado en 2017, que aspira a formar en cuatro años a 100.000 programadores, 10.000 profesionales y 1.000 emprendedores.

La alta demanda de trabajadores conduce a que muchos estudiantes universitarios de las carreras afines a sistemas se inicien laboralmente antes de titularse, e incluso muchos las abandonen. Incide en ello, además de la dificultad de combinar el trabajo y el estudio, la falta de relevancia que tiene el título en términos salariales en el sector. Así, resulta un rasgo particular del mundo laboral del SSI la valoración del saber hacer de los trabajadores por encima de sus credenciales educativas, basados en la evaluación de su experiencia en el manejo de tecnologías que pueden darse no sólo en instituciones educativas sino también en cursos y capacitaciones, y de forma autodidacta (Dughera et al., 2012).

Sin embargo, a pesar de la alta demanda de trabajadores, los salarios de los trabajadores resultan un aspecto problemático en el sector, caracterizado por su heterogeneidad y una incidencia de bajas 
remuneraciones en algunos de los puestos laborales de menor jerarquía (Murmis, 2015; Ferpozzi y Zukerfeld, 2012). Al respecto Andrés Rabosto y Mariano Zukerfeld (2017) señalan que ello contradice el principio de la teoría económica liberal donde ante una situación de mercado con alta demanda y baja oferta crece el valor de la mercancía, en este caso, de la fuerza de trabajo. A diferencia de la cantidad de empresas, ventas, exportaciones y empleo, los salarios del sector de SSI no crecieron en sintonía, e incluso en muchos casos se encuentran en sus valores por debajo de los de otros sectores productivos (que acceden a actualizaciones salariales periódicas a partir de la realización de paritarias).

Si bien desarrollaremos con mayor profundidad este aspecto en el apartado de análisis de la precariedad laboral podemos adelantar que inciden en ello factores que exceden a la situación individual de cada trabajador, como la ausencia de un sindicato reconocido con personería gremial que negocie colectivamente los salarios y de un CCT que establezca bandas salariales. Esta situación desregulada deja lugar a la negociación individual de los trabajadores y a la aplicación de criterios empresariales discrecionales para la determinación de las remuneraciones (como el desempeño o la capacidad), segmentando así las condiciones laborales.

$\mathrm{Al}$ respecto, Montes Cató (2010) advierte como rasgo característico y funcional al desarrollo del sector de SSI un proceso articulado de deslaboralización e individualización de las relaciones laborales, que refieren respectivamente a la falta de reconocimiento de la relación de dependencia entre la empresa y el trabajador (ante la multiplicación de formas de contratación como prestación de servicios, consultoría, tercerización laboral) y al desarrollo de negociaciones personales sobre jornadas y salarios. Esto se complementa, además, con el despliegue de dispositivos empresariales antisindicales que desalientan la organización colectiva de los informáticos, legitimados por la falta de reconocimiento de la personería gremial a algún sindicato del sector, que mantienen el status quo de segmentación y desregulación laboral.

\section{Ambivalencias laborales de un gigante productivo: precariedad laboral en el sector de SSI}

El abordaje de las condiciones laborales en el sector de SSI ha tenido aportes en los últimos años por parte de estudios que focalizaron su análisis en alguna de sus problemáticas particulares. Al respecto, identificamos tres orientaciones de abordaje: en primer lugar, Pablo Míguez (2017) se focalizó en los efectos que genera la subcontratación creciente en el sector en términos de desprotección e inestabilidad laboral; en segundo lugar, Juan Montes Cató (2010, 2011), y recientemente Hernán Palermo (2018), ponen luz sobre el proceso de deslaboralización e individualización laboral característico del sector y en sus efectos sobre la intensidad del trabajo y la inestabilidad laboral; mientras que, en tercer lugar, el equipo de Mariano Zukerfeld (2012; Ferpozzi y Zukerfeld, 2012; Rabosto y Zukerfeld, 2017) y Juan Ignacio Del Fueyo (2017), lo hacen 
en torno a la ausencia de un de un CCT y un sindicato reconocido oficialmente, y sus efectos sobre la degradación e individualización de los salarios de los trabajadores informáticos.

Nuestra propuesta consiste en realizar un análisis de contenido de esos y otros estudios referidos a las condiciones de trabajo en el sector de SSI, a partir de una concepción ampliada de la precariedad laboral, poniendo en diálogo sus perspectivas particulares sobre las problemáticas del sector. Esto implica su codificación ${ }^{7}$ a partir del abordaje de las dimensiones contractuales, subjetivas y relacionales que la componen. Se trata por tanto de una propuesta de análisis de dichos trabajos empíricos, realizados por cientistas sociales contemporáneos, a la luz de nuestro instrumento teórico multidimensional de abordaje de la precariedad laboral.

\section{Dimensión contractual}

El sector SSI se caracteriza en términos contractuales por una alta incidencia de formas laborales asalariadas y registradas. Incluso, resulta una de las ramas donde más creció el empleo registrado en los últimos años (Zukerfeld, 2012). Aunque también, de forma paralela, se dio un importante crecimiento en las formas de contratación laboral flexible, bajo la modalidad freelance, contratación por servicios y otras formas de subcontratación laboral (Miguez, 2017). Si bien no se puede establecer con exactitud el número y la evolución de estas formas laborales, desde el OPSSI, realizaron una estimación que considera que por cada 10 trabajadores registrados habría uno no registrado en el sector (OPSSI, 2018).

$\mathrm{Al}$ respecto, Montes Cató (2010) advierte acerca del subconjunto de trabajadores informáticos contratados de forma flexible que, a diferencia de los asalariados formales, priman los principios de inestabilidad laboral y desprotección sindical. Asocia esto al proceso de deslaboralización, que consiste en el corrimiento de las figuras clásicas asociadas al derecho de trabajo a través de formas de contratación novedosas (como el contrato por obra y la prestación de servicios) que desdibujan la relación de dependencia entre el trabajador y la empresa, equiparándolos como en una supuesta relación de intercambio comercial.

Por su parte, Miguez $(2010,2016)$ aporta la incidencia que tiene en el sector de SSI la tercerización de actividades laborales a través de la subcontratación de trabajadores, que conduce a una precarización en sus condiciones laborales, fundamentalmente en términos salariales. La tercerización se ve facilitada por la naturaleza del producto/servicio y por las condiciones técnicas del sector informático que habilitan su división en etapas (y su distribución) y, a diferencia de otros sectores, no necesariamente responde a trabajos de bajas calificaciones ${ }^{8}$.

Así se da muchas veces que las empresas de SSI subcontratan de forma temporal a especialistas en función a la demanda del proyecto en que se encuentran trabajando. Muchos de estos trabajadores contratados temporalmente trabajan desde su casa, bajo la modalidad freelance. Al respecto, Paula Lenguita (2010) señala que muchos trabajadores 
informáticos valoran positivamente esta forma de trabajo independiente por los beneficios en cuanto a su flexibilidad en torno al manejo horario y lugar de trabajo, y la posibilidad de, en ocasiones, cobrar mayores ingresos (fundamentalmente con clientes extranjeros). Aunque remarca que esto tiene como contrapartida la inestabilidad y la ausencia de protecciones laborales propias de una relación de trabajo asalariada (aportes jubilatorios, obra social, vacaciones, licencias, asignaciones familiares, indemnización por despido, etc.).

\section{Dimensión subjetiva}

El abordaje de dimensión subjetiva de la precariedad laboral nos revela las insatisfacciones de los trabajadores respecto a sus formas de reconocimiento simbólico y material en el trabajo. Se focaliza así en sus percepciones sobre las formas de organización del trabajo y de sus tareas (en su carácter simbólico) y sobre las formas de retribución salarial (en su carácter material). En términos metodológicos, si bien algunos cuestionarios de encuestas en relevamientos estadísticos permiten acceder a las insatisfacciones de trabajadores (referidas a la búsqueda de otro empleo estando ocupado, riesgos psicosociales en el trabajo o demandas en conflictos sindicales), resultan principalmente las técnicas cualitativas las más utilizadas para su abordaje por su pertinencia para acceder en profundidad al universo subjetivo de los sujetos (Vasilachis, 2008).

A través del análisis de contenido de los estudios recientes del sector de SSI que aplicaron instrumentos metodológicos cualitativos de medición (entrevistas principalmente), encontramos que una problemática laboral que emerge al analizar las insatisfacciones de los TI es la intensidad de su trabajo, la cual refiere a la realización de tareas laborales durante largas jornadas y a un alto ritmo. Esta situación se da especialmente en situaciones de cierre de proyectos y entregas de productos/servicios a clientes, que resultan frecuentes en la forma de organización del trabajo por objetivos, en función a demandas concretas y en plazos cortos de trabajo (Montes Cató, 2010). Se habla entonces, dentro de la jerga de los TI de que el proyecto se quema, y es necesario redoblar los esfuerzos y las jornadas de trabajo para resolverlo. Al respecto, Palermo (2018) marca el desgaste mental que este ritmo de trabajo genera en términos de salud en los trabajadores. Esto implica muchas veces trabajar horas extras, que incluyen madrugadas y fines de semana, los cuales no siempre son recompensados económicamente.

Por su parte Gerald Reischl (2008) contribuye a su comprensión al señalar que en la industria del software se combina la comodidad con la creación y la producción en un ámbito que prologa las horas laborales. Se refiere con ella al modelo de oficinas google, que resulta un emblema como lugar de trabajo del sector de SSI y es emulado por la mayoría de las empresas de software a nivel mundial. Se trata de espacios laborales caracterizados como lugares desestructurados y lúdicos, que simulan el confort de un hogar y de sus elementos de ocio, y actúan como 
catalizadores de las largas jornadas, habilitando a su vez su prolongación (Zukerfled, 2012).

Pero además de las fechas de entregas y de cierres de proyectos, la propia cotidianidad del trabajo informático conduce a que muchos trabajadores sigan trabajando más allá de la jornada laboral, para resolver tareas que quedaron pendientes o para actualizarse en el manejo de tecnologías. Para ello recurren a portales y blogs de especialistas del sector donde se comparten experiencias y respuestas a problemáticas informáticas. Esas búsquedas y experiencias de autoformación se realizan la mayoría de las veces durante el tiempo libre, tras la jornada de trabajo. Al respecto, Montes Cató (2010) señala la existencia de una constante exigencia de capacitación y formación en el área de programación, que de no hacerla implicaría quedar afuera del mercado laboral y genera angustia en los trabajadores. $<<$ Muchas veces se trata de correr más rápido (capacitarse más) para estar en el mismo lugar, es decir mantener lo que se tiene pero con mayor esfuerzo >> (Montes Cató, 2010, p. 82).

Esta intensidad y prolongación del tiempo de trabajo es la contratara de la flexibilidad horaria laboral, que es señalada desde ámbitos empresariales y oficiales como uno de los aspectos positivos del trabajo en el marco de la sociedad post-industrial que, gracias a las nuevas tecnologías de comunicación en el trabajo, derriba fronteras espaciales y temporales propiciando la autonomía del trabajador en la administración del tiempo y lugar de trabajo. En el caso de los TI, estos cuentan con la posibilidad de auto-administrarse recreos durante la jornada de trabajo (en muchas ocasiones, utilizando los elementos de dispersión provistos por la empresa como televisores, juegos, instrumentos musicales, etc.), trabajar desde la casa o incluso entrar un poco más tarde del horario convenido. Lo cual es señalado por ellos como uno de los aspectos más positivos y satisfactorios de sus tareas (Lenguita, 2010)

Sin embargo, la compensación implícita de esa flexibilidad está en la posibilidad de extender la propia jornada de trabajo cuando el proyecto o la empresa lo requieran. Y si bien el trabajo extra jornada resulta un rasgo compartido con otros sectores productivos, la particularidad del SSI es su justificación empresarial en relación a la vocación y el interés del trabajador informático, a la que presenta también como un hobby. Juega en esa fundamentación otro tipo de dispositivo de deslaboralización, ya no en términos contractuales ni espaciales, sino de la propia tarea, la cual es considerada en una zona gris con el propio disfrute del trabajador (Palermo, 2018).

Pero es fundamentalmente en torno a la falta de reconocimiento material donde los estudios del sector de SSI coinciden en señalar la principal fuente de insatisfacción de los trabajadores informáticos, expresado en bajos salarios y ausencia actualizaciones salariales. Esa insatisfacción emerge como indicador compartido en las constantes demandas de aumentos salariales de parte de los distintos sindicatos del sector, y como el principal motivo de rotación laboral (Montes Cató, 2010; Murmis, 2015; Segura y otros, 2012; Zukerfeld, 2013). 
$\mathrm{Al}$ respecto, como marco contextual de dichas insatisfacciones, Andrés Rabosto y Mariano Zukerfeld (2017) mostraron cómo entre 1998 y 2014 los trabajadores del sector de SSI fueron los únicos que en vez de aumentar sus salarios reales tuvieron un retroceso en los mismos. Incide en ello la falta de un sindicato reconocido que negocie un CCT específico para el sector, que estipule bandas salariales y mecanismos de actualización salarial (paritarias generales y regulares). Así los salarios del sector de SSI se encuentran totalmente des-regulados, cada empresa define los mismos de forma arbitraria y negociándolos de forma individual con cada trabajador, lo cual da lugar una situación salarial segmentada.

\section{Dimensión relaciones de trabajo}

El abordaje de la dimensión relacional de la precariedad implica ampliar nuestra unidad de observación de su carácter individual a otra de tipo colectivo. En las anteriores dimensiones abordamos al trabajador en su unicidad (su contrato y su subjetividad), mientras que ahora lo miramos como parte de un colectivo de trabajadores en relaciones de fuerza (desiguales) frente al capital. Sobre esta relación de poder, constitutiva del mundo laboral capitalista, focaliza la mirada la perspectiva neomarxista (Béroud y Bouffartigue, 2009 y Cingolani, 2009) que incorpora al análisis de la precariedad el estudio del debilitamiento de los derechos sindicales.

La precarización de relaciones laborales se expresa en la degradación de las formas de organización y representación colectiva de los trabajadores, tanto en su carácter formal (ausencia de derecho a la afiliación sindical, ausencia de un sindicato reconocido) como de hecho (ausencia de representación real por parte del sindicato, prácticas empresariales antisindicales, entre otras). Tiene como consecuencia, por tanto, la dificultad de los trabajadores para organizarse y desarrollar reclamos por la mejora de las condiciones laborales.

En el caso del sector del software y servicios informáticos, observamos que la precarización en sus relaciones laborales se da, en primer lugar, por la ausencia de un sindicato del sector reconocido (en su personería gremial $)^{9}$. Esta ausencia inhabilita su participación en instancias de negociaciones colectivas y también la celebración de un CCT, que regule y normativice las condiciones de trabajo en el sector de SSI. De esta manera, entendemos que la dimensión relacional de la precarización laboral asume un carácter explicativo de gran parte de la degradación de las dimensiones contractuales y subjetivas antes mencionadas, al habilitar la discrecionalidad empresarial e individualización en las condiciones contractuales, salariales y materiales de trabajo (jornada y ritmo de trabajo), que segmentan y debilitan al colectivo de trabajadores informáticos.

Actualmente existen cuatro sindicatos nacionales en el sector de SSI que se disputan el reconocimiento de su personería gremial: Unión Informática (UI), Asociación Gremial de Computación (AGC), Unión de Trabajadores Sociedad de Autores (UTSA) y Sindicato Único de Trabajadores Informáticos de la República Argentina (SUTIRA). Todas 
estas agrupaciones sindicales se encuentran inscriptas jurídicamente pero carecen de la personería ${ }^{10}$ que les permita la participación en paritarias, negociaciones colectivas y la elaboración de un CCT para el sector. Si bien los cuatro sindicatos representan formalmente a TI a partir de su afiliación sindical, la participación en reclamos puntuales ${ }^{11}$ y la celebración de convenios por empresa ${ }^{12}$, se trata de una representación fragmentada que no abarca al conjunto de los trabajadores del sector, ni en sus prácticas ni en sus resultados.

$\mathrm{Al}$ respecto, Montes Cato (2010) habla de un proceso de individualización en el sector de SSI, que junto con la deslaboralización, actúan como dispositivos de control sobre los trabajadores informáticos. El dispositivo de individualización, legitimado por la ausencia de un CCT que establezca la aplicación del derecho colectivo de trabajo, se refuerza con la ausencia de un sindicato reconocido que habilite el desarrollo de procesos de colectivización de demandas y negociaciones. Este debilitamiento en los derechos sindicales de los trabajadores informáticos, se ve reforzado por prácticas empresariales antisindicales, como el hostigamiento a delegados y hasta la prohibición al accionar sindical dentro de los lugares laborales (Montes Cató, 2010), que desalienta la organización colectiva.

\section{Reflexiones finales}

La consolidación de la sociedad post-industrial implicó una nueva forma de organización del trabajo basado en el uso intensivo de las tecnologías de comunicación y el conocimiento que desterritorializaron la producción, abriendo la posibilidad de una globalización productiva que aceleró tiempos de trabajo y acortó distancias. Frente al esquema tradicional industrial de producciones materiales en masa, localizadas en grandes emplazamientos productivos localizados y trabajadores estables realizando tareas reiterativas, el nuevo modelo productivo habilita una fábrica difusa que se emplaza en diferentes (y lejanos) lugares productivos articulados a partir de las nuevas tecnologías de comunicación, con aplicación e inversión en el desarrollo del conocimiento y la búsqueda de la diferenciación (y distinción) en la producción, especialmente, de bienes inmateriales.

El sector de software y servicios informáticos aparece como emblema de esta nueva etapa productiva, atravesado por rasgos de desterritoralización productiva, uso intensivo de la tecnología y del conocimiento, y producción de bienes inmateriales. Pero a pesar de que estas transformaciones productivas habilitaron un aceleramiento de la producción y una integración global, reduciendo distancias y tiempos de trabajo, tienen como contracara la desprotección e inestabilidad en las condiciones laborales de los trabajadores post-industriales. Éstas se amparan en el proceso de flexibilización laboral que acompaña los nuevos tiempos productivos, caracterizados por la subcontratación, 
intensificación del ritmo del trabajo, contratos temporales, segmentación laboral, entre otros.

En el caso del sector de SSI en Argentina vemos que el crecimiento productivo exponencial y comercial no se refleja de la misma manera en la calidad de las condiciones laborales de los trabajadores informáticos, que se encuentran desreguladas y segmentadas (especialmente en términos salariales) por la ausencia de un CCT. Esto se da a pesar de la alta incidencia del empleo registrado en el sector, que es uno de los que más creció en ese sentido. A partir de esto y buscando dar luz al proceso de degradación laboral que atraviesa el sector de SSI, aplicamos como instrumento de análisis nuestra concepción ampliada de la precarización laboral para acceder no sólo a sus condiciones contractuales sino también a las subjetivas y de relaciones laborales. Utilizamos como insumo investigaciones sociales recientes que focalizaron su estudio de alguna de dichas dimensiones en el sector de SSI, proponiendo un diálogo entre ellas.

Así encontramos que en la propia legalidad del sistema que regula los contratos laborales de SSI se habilita el uso de figuras deslaboralizadas (como monotributista, prestador de servicios, trabajador freelance) que muchas veces encubren la relación de dependencia de los trabajadores con su empleador, privándolos del acceso a protecciones y estabilidades laborales fundamentales. Por otro lado, el abordaje de las insatisfacciones de los informáticos reveló como problemáticas compartidas la intensidad del trabajo y sus bajos salarios. Por último, al analizar sus relaciones laborales, encontramos que a pesar de que los informáticos cuentan con el derecho formal a la sindicalización, éste no se aplica totalmente en los hechos, ya que son objeto de prácticas de hostigamiento sindical empresarial y aún permanece vacante el reconocimiento de la personería gremial que habilite y el desarrollo de negociaciones colectivas.

Muchas de estas problemáticas habían sido señaladas de forma segmentada por diferentes investigaciones académicas sobre el sector de SSI, y en la mayoría de los casos sin categorizar a las mismas como parte de un proceso de precarización laboral. Nuestro aporte consistió en realizar una lectura integrada y comparativa de las mismas bajo un concepto ampliado de precarización laboral, para describir el proceso de degradación de las condiciones laborales tomando en consideración no solo el tipo de contrato laboral, sino también las insatisfacciones de los trabajadores y la débil aplicación de sus derechos sindicales.

También pudimos analizar las implicancias recíprocas entre las diferentes dimensiones que conforman la degradación de la calidad del trabajo. Así, observamos que la débil aplicación del derecho colectivo de trabajo se ve afectado por la ausencia de un sindicato del sector reconocido que celebre un CCT y regule colectivamente las condiciones de contratación, retribución salarial y organización del tiempo de trabajo. Por otro lado, la débil aplicación de los derechos sindicales limita sus posibilidades de realizar acciones colectivas para la transformación de las degradaciones que afectan sus contratos y sus subjetividades. 
Como reflexión final, y a partir del sector de SSI como caso instrumental de abordaje de la sociedad post-industrial, pudimos observar que a pesar del cambio productivo, tras la crisis del modelo fordista, siguen vigentes problemáticas laborales históricas como la inestabilidad y desprotección laboral. Las cuales se profundizan ante la propagación y legalización de nuevas formas contractuales temporales, la intensificación del ritmo del trabajo por el uso de nuevas tecnología y la segmentación de condiciones laborales, salariales e instatisfacciones. Consideramos que esta complejización del mundo laboral post-industrial exige de parte de nuestros instrumentos teóricos y metodológicos de investigación una apertura multidimensional que nos permita visibilizar las nuevas formas que adopta el proceso ofensivo sobre los trabajadores, y también sus resistencias.

\section{Referencias}

Adamini, M. (2014). Formaciones identitarias en lugares de trabajo precario: Un estudio sobre pasantes de la administración pública de la provincia de Buenos Aires (2008-2012). (Tesis de Doctorado en Ciencias Sociales). Facultad de Humanidades y Ciencias de la Educación, UNLP, La Plata. En Memoria Académica. Disponible en: http:// www.memoria.fahce.unlp.edu.ar/tesis/te.1014/te.1014.pdf

Appay, B., (2005), La dictature du succès. Le paradoxe de l'autonomie contrôlée et de la précarisation. Paris: L'Harmattan.

Azpiazu, D., Basualdo, E. y Nochteff, H. (1990). Los límites de las políticas industriales en un período de reestructuración regresiva: el caso de la informática en Argentina. Desarrollo económico, vol. 30, No 118.

Babini, R. (2003). La Argentina y la computadora. Crónica de una frustración. Buenos Aires: Ed. Dunken.

Battistini, O. (2009). La precariedad como referencial identitario. Un estudio sobre la realidad del trabajo en la Argentina actual. Psicoperspectivas, VIII (2), pp.120-142. Recuperado de: https://www.psicoperspectivas.cl/ index.php/psicoperspectivas/article/viewFile/65/74

Bauman, Z., (2000). Modernidad Líquida. Buenos Aires: FCE.

Bekerman, M; y Cataife, G. (2001). Encadenamientos productivos: estilización e impactos sobre el desarrollo de los países periféricos. Anales de la Asociación Argentina de Economía Politica.

Boutang, Y. M. (1999) Riqueza, propiedad, libertad y renta en el capitalismo cognitivo. En Rodríguez, E. y Sánchez, R. (Comp.) Capitalismo cognitivo, propiedad intelectual y creación colectiva. Madrid: Traficantes de Sueños.

Berelson, B. (1952). Content Analysis in Comunication Research. Glencoe: Free Press.

Béroud, S. y Bouffartigue, P. (Comp.) (2009). Quand le travail se précarise, quelles résistances collectives? París: La dispute.

Borello, J.; Erbes, A.; Robert, V. Roitter, S.y Yoguel, G. (2005). Competencias técnicas de los trabajadores informáticos. El caso de la Argentina. Revista de la CEPAL, No. 87, pp. 131-150. Recuperado de: https:// repositorio.cepal.org/handle/11362/11084 
Bulloni, M.N. (2013) La organización productiva y del trabajo en las redes de proyectos del cine publicitario. Papeles de Trabajo, 12, pp. 9-19. Recuperado de: http://www.idaes.edu.ar/papelesdetrabajo/ paginas/Documentos/n12/02_DOS_Bulloni.pdf

Castel R. (1997). La metamorfosis de la cuestión social. Una crónica del salariado. Buenos Aires: Paidós.

Cavarozzi, M. (1996) El capitalismo político tardío y su crisis en América Latina. Rosario: Homo Sapiens Ediciones.

Cingolani P. (2009). Ce qu'il y a de nouveau dans le travail précaire. Ouvrir laréflexion savante sur le questionnement politique. En Beroud S. y Bouffartigue P. (Dir.) Quand le travail se précarise, quelles résistances collectives? París: La Dispute.

De la Garza, E. (2010). Hacia un concepto ampliado de trabajo. Del concepto clásico al no clásico, México, Anthropos/uam-Iztapalapa. Cuadernos A, Temas de Innovación Social, 33.

Del Fueyo, J. I. (2017). Las prácticas sociales sindicales en la industria Informática Argentina. Ponencia presentada en $13^{\circ}$ Congreso Nacional de Estudios del Trabajo, Buenos Aires. Recuperado de: https:// www.aset.org.ar/2017/ponencias/15_DelFueyo.pdf

Diana Menéndez, N. (2007). La representación sindical en el Estado: los casos de la Asociación de Trabajadores del Estado (ATE) y la Unión del Personal Civil de la Nación (UPCN). (Tesis de Maestría en Ciencias Sociales inédita). Facultad de Ciencias Sociales, UBA, Buenos Aires.

Diana Menéndez, N. (2009). Condiciones de trabajo y precarización laboral en el Estado. El caso de los trabajadores del Ministerio de Trabajo, Empleo y Seguridad Social de la Nación. (Tesis de Doctorado en Ciencias Sociales inédita). Facultad de Ciencias Sociales, UBA, Buenos Aires.

Dubar, C. (2002). Las crisis de las identidades: Lainterpretación de una mutación, Ediciones Bellaterra, Barcelona.

Dughera, L.; Ferpozzi, H.; Gajst, N.; Mura, N.; Yannoulas, M.; Yansen, G.; Zukerfeld, M. (2012). Una aproximación al subsector del Software y Servicios Informáticos (SSI) y las políticas públicas en la Argentina. $41^{\circ}$ JAIIO-SSI, $10^{\circ}$ Simposio sobre la Sociedad de la Información, pp. 187-209.

Ferpozzi, H. y Zukerfeld, M. (2012). El sindicalismo en el trabajo informacional: un análisis de la relación entre los trabajadores informáticos y el mundo gremial. Ponencia presentada en el $I V$ Congreso Anual de AEDA, Buenos Aires. Recuperado de: http://e-tcs.org/wp-content/uploads/2012/08/Ponencia-FerpozziZukerfeld-Trabajo-informacional-y-sindicalismo-AEDA-2012.pdf

Garretón, M. A. (2002). La transformación de la acción colectiva en América Latina. Revista de la CEPAL, No 76, pp. 7-24.

Henry, L. (2013). Flexibilización productiva y difusión del trabajo independiente en la prensa escrita argentina: un estudio sobre las condiciones de empleo de los periodistas colaboradores. Trabajo $y$ Sociedad, 21, pp. 261-278. Recuperado de: http://www.unse.edu.ar/ trabajoysociedad/21\%20HENRY\%20flexibilacion\%20productiva \%20trabajo\%20independiente.pdf

Lenguita, P. (2010). Las relaciones de teletrabajo: Entre la protección y la reforma. Argumentos, año 23, núm. 64, pp. 245-263. 
Longo, J. (2014). ¿Renovación de las tradiciones sindicales en ámbitos laborales precarizados?: un análisis de las organizaciones sindicales en empresas supermercadistas durante la posconvertibilidad. (Tesis de Doctorado en Ciencias Sociales inédita). Facultad de Ciencias Sociales, UBA., Buenos Aires.

Longo, J. y Busso, M. (2017). Precariedades: Sus heterogeneidades e implicancias en el empleo de los jóvenes en Argentina. Estudios del Trabajo, Núm. 53.

López, A. y Ramos, S., (2008). La industria de software y servicios informáticos argentina. Tendencias, factores de competitividad y clusters, CENIT.

López, A.y Ramos, D. (2009), "Oportunidades y desafíos para la industria del software en Argentina", en Bastos Tigre, P. y Silveira Marques, F. (ed.). Desafios y oportunidades para la industria del software en América Latina, CEPAL, Naciones Unidas.

López, A. y Ramos, D. (2013). “Análisis de diagnóstico tecnológico sectorial: Software y servicios informáticos", Informe del MINCYT. Recuperado de: https://www.argentina.gob.ar/ciencia/publicaciones.

Míguez, P. (2010). Automatización y revolución informática. Los cambios en el proceso de trabajo y sus efectos en el trabajo. En Montes Cató, J. (coord) El trabajo en el capitalismo informacional. Los trabajadores de la industria del software. Benavídez: Poder y trabajo editores.

Míguez, P. (2017) Trabajo, conocimiento y precariedad laboral en el capitalismo contemporáneo. Revista Ciencias Sociales. Universidad de Buenos Aires.

Ministerio de Hacienda y Finanzas Públicas Presidencia de la Nación (2016). Informes de Cadena de Valor Software y servicios informáticos, Año 1, N ${ }^{\circ} 12$, Buenos Aires.

Mochi Alemán, P.O. (2002), El movimiento del software libre. Revista Mexicana de Ciencias Politicas y Sociales, vol. XLV, núm. 185, pp. 73-89.

Montes Cató, J. (coord) (2010) El trabajo en el capitalismo informacional. Los trabajadores de la industria del software. Benavídez: Poder y trabajo editores.

Montes Cató, J. (2011). El trabajo en la sociedad de la información: desafíos para el movimiento obrero; Universidad Nacional de San Luis; Kairos, (27), pp. 15-35.

Moulier-Boutang Y. (1998). De l'esclavage au salariat. Economie historique du salariat bridé. París: PUF-Actuel Marx.

Murmis, E. (2015). En la tecla. Condiciones laborales y organización de los trabajadores informáticos. El Aromo. (76).

Nahirñak, P. (2006). Los primeros resultados de la Ley del Software. Una industria que avanza a paso firme pero que puede dar mucho más. Revista Novedades, IERAL, Fundación Mediterránea.

Novik M., Roitter S., Yoguel G., Borello J.y Milesi D., (2004). Información y conocimiento: la difusión de TIC \#s en la industria manufacturera argentina, Revista de la CEPAL, No82, pp. 139-156.

Observatorio Permanente de la Industria de Software y Servicios Informáticos (2008). Situación y perspectivas de las PyMES del sector del SSI en la Argentina. Buenos Aires: CESSI. Recuperado de: http://www.observatoriopyme.org.ar/newsite/wpcontent/uploads/2014/09/FOP_IC_0806_Informe-2008.-Situacion-yperspectiva-de-PSSI.pdf 
Observatorio Permanente de la Industria de Software y Servicios Informáticos (2018). Reporte anual sobre el Sector de Software y Servicios Informáticos de la República Argentina. Buenos Aires: CESSI.

Palermo, H. (2018), Masculinidades en la industria del softwareen Argentina. Revista Internacional de Organizaciones, No 20, pp. 103-121. Recuperado de: http://www.revista-rio.org/index.php/ revista_rio/article/view/254/223

Paugam, S. (2000) Le salarié de la précarité. Paris: PUF.

Pok, C. (1992), Precariedad laboral: personificaciones sociales en la frontera de la estructura del empleo. Documento de trabajo. Seminario Interamericano de Medición del Sector Informal, COM-CIE, OEA-INEI.

Reischl, G. (2008). El engaño Google: una potencia mundial incontrolada en internet. Barcelona: Medialive Content.

Rabosto, A. y Zukerfeld, M. (2017) Precarity, precariousness and software workers Wages, unions and subjectivity in the Argentinian Software and Information Services sector, Journal Work Organization, Labour and Globalisation, Vol. 11, pp. 87 - 102

Sennett, R. (2000). La corrosión del carácter. Las consecuencias personales del trabajo en el nuevo capitalismo. Barcelona: Anagrama.

Strauss, A. y Corbin, J. (2002). Bases de la investigación cualitativa. Técnicas y procedimientos para desarrollar la teoría fundamentada. Medellín: Editorial Universidad de Antioquia.

Vasilachis de Gialdino, I. (2008), "Los fundamentos epistemológicos de la investigación cualitativa" en N. Cohen \& I. Piovani (Comp.) La metodología de la investigación en debate, Eudeba- Ed. UNLP, Buenos Aires.

Zukerfeld, M. (2012) Una introducción al Sector información y al Trabajo informacional. En Dughera, L.; Yansen, G.; Zukerfeld, M., (eds.) Gente con códigos. La heterogeneidad de los procesosproductivos de software. Buenos Aires: Universidad Maimónides.

\section{Notas}

1 La condición de no registro de la relación laboral (representada por la falta de aportes a la seguridad social) es el principal indicador de medición estadístico de la precariedad laboral por su condición de variable proxy de ausencia de otros derechos laborales fundamentales de una relación laboral estable y protegida como son la obra social, las asignaciones familiares, la indemnización por despido, licencias, etc.

2 Esta ley se orienta a aquellas empresas que realicen actividades de investigación y desarrollo, apliquen normas de calidad en la producción o realicen exportaciones.

3 Recientemente, en mayo de 2019 se sancionó en el Congreso la aprobación una nueva legislación, titulada Ley de la Economía del Conocimiento, que reemplaza la anterior y prolonga hasta 2030 el marco impositivo especial para las empresas del sector de SSI y también para las de biotecnología, ingeniería, servicios profesionales y producciones audiovisuales (nuevo sectores incorporados).

4 Aunque en 2018, dicha subsecretaría fue eliminada, en el marco del plan de recorte estatal y reordenamiento ministerial, pasando a ser una dirección de la Secretaría de Emprendedores y Pyme (Sepyme). 
5 Sin embargo, a pesar de este crecimiento en el perfil exportador, a nivel regional Argentina se encuentra en un nivel inferior en comparación con Brasil, en primer lugar, y México, en segundo lugar. A nivel internacional, Argentina ocupa el puesto 21, siendo el principal exportador es India, con filiales de empresas extranjeras atraídas por los bajos niveles salariales de trabajadores capacitados. De todas maneras, progresivamente, el país va ganando un mejor posicionamiento en el comercio internacional (Ministerio de Hacienda y Finanzas Públicas Presidencia de la Nación, 2016).

6 Este dato resulta una estimación realizada por el OPSSI a partir de considerar que por cada 10 trabajadores registrados habría uno no registrado en el sector (OPSSI, 2018).

7 La codificación resulta un proceso analítico por medio del cual se fragmentan, conceptualizan e integran los datos para formar una teoría (Strauss y Corbin, 2002).

8 Lopez y Ramon (2013) marcan que los límites a la subcontratación en el sector de SSI se dan en actividades donde no existe la posibilidad de que el conocimiento producido se convierta en insumo de ese trabajador como posible competidor. Por ese motivo, señalan que la tercerización resulta mayoritaria en las etapas más rutinarias del proceso de trabajo (diseño, codificación, testeo, soporte), que no dejan por ello de ser actividades calificadas que requieren conocimientos específicos por parte de los trabajadores. Lo cual lo diferencia de otros sectores productivos donde se tercerizan las tareas más rutinarias pueden ser desarrolladas por personal no calificado.

$9 \mathrm{Al}$ momento de escribir este artículo (junio de 2019), desde la página web de la Asociación Gremial de Computación (AGC) se indicaba que el sindicato había obtenido personería gremial para la representación de los trabajadores que desarrollen tareas de operador, programador, analista soporte técnico, desarrollo de sistemas, centro de cómputos, técnicos, con zona de actuación en el territorio de la Ciudad Autónoma de Buenos Aires y Provincia de Buenos Aires. La misma fue otorgada en noviembre de 2018, a partir de la resolución 239/2018, por parte de la Secretaría de Gobierno de Empleo y Trabajo, a cargo de Jorge Triaca. Sin embargo, dicha regulación aún no fue publicada oficialmente por el boletín oficial ni cuenta con su inscripción registral, requisito para su efectivización.

10 La determinación de la personería es definida por el MTEySS (actualmente Secretaría de Gobierno de Trabajo y Empleo) en función de aquellas asociaciones gremiales que resulten las más representativas al interior de la rama o actividad, afiliando a por lo menos al $20 \%$ del colectivo que aspiran a representar. A pesar de ello, algunos sindicatos participaron de la elaboración de convenios por empresa.

11 En el caso de UI cuenta con un importante activismo de base, que se cristaliza en diferentes conflictos por reclamos, que incluyen movilizaciones y paro de actividades. Entre ellos se destaca su conflicto inaugural en 2011 por la mejora de las condiciones salariales y posteriores despidos en IBM, y posteriormente en empresas como SONDA, Avaya, NGA, Unys, NCR, Neuralsoft (Rosario), Tecnoazar, Stefanini e Indra (Del Fueyo, 2017). El resto de los sindicatos tienen una tendencia menos combativa y más proclive a la negociación con los empresarios como estrategia de resolución de reclamos y problemáticas. Sin embargo, se destaca que AGC en 2016 participó de movilizaciones por el despido de informáticos de HP, Conectar Igualdad y ATOS; y SUTIRA en 2015 movilizó por la apertura de paritarias.

12 Todos los sindicatos del sector elaboraron un modelo de CCT, algunos de los cuales fueron firmados por empresa, pero ninguno logró la homologación del MTEySS para su extensión a todos los trabajadores del sector, para lo cual se requiere la personería gremial que habilite su negociación colectiva como marco de su celebración. En el caso de UI elaboró en 2015 un CCT, que fue aplicado en 11 empresas (Del Fueyo, 2017), la mayoría de grandes ciudades. 
Marina Adamini. Una mirada ampliada sobre la precariedad post-industrial. El caso de los trabajadores informáticos a partir de estudios soci...

Por otra parte, el resto de los sindicatos manifiesta haber firmado un CCT con la CESSI (aunque ninguno fue homologado por el MTEySS): AGC en 1994, UTSA en 2013 y SUTIRA en 2013 (aunque éste último es negado por la CESSI). 